\title{
JORNADAS DE AUTO Y MUTUA CAPACITACIÓN CON LOS MAESTROS Y LAS MAESTRAS DE LENGUA Y CULTURA DEL DISTRITO DE CHIRRIPÓ
}

\author{
Hannia Watson' \\ Lolita Camacho ${ }^{2}$ \\ Cristian Brenes ${ }^{3}$
}

Resumen

Con el propósito de contribuir a la disminución de las desventajas educativas en que se han desarrollado las comunidades cabécares del distrito de Chirripó, se planea el Proyecto Jornadas de auto y mutua capacitación con los maestros y las maestras de Lengua y Cultura, como una estrategia para mejorar la calidad de la práctica docente y generar procesos educativos culturalmente pertinentes.

Palabras claves: procesos educativos, interculturalidad, pertinencia cultural, práctica docente.

\section{Abstract}

The author presents the Project "Journeys of auto and mutual training in Chirripo District" that were developed with mother tongue and culture teachers in Cabecares communities to diminish the educative gaps that these communities have. The project brings a strategy to improve the quality of teaching practices and generates culturally pertinent educational processes.

Keywords: educational processes, intercultural, cultural pertinence, teaching practice.

\section{Introducción}

$\mathrm{E}$ n el año 2004, la sede del Atlántico de la Universidad de Costa Rica inició un proceso de reflexión en torno al acceso a la educación superior

\footnotetext{
MEd. Profesora Sede del Atlántico UCR hwatson@sa.ucr.ac.cr Licda. Profesora Sede del Atlántico UCR Icamacho@sa.ucr.ac.cr MSc. Profesor Sede del Atlántico UCR cbrenes@cariari.ucr.ac.cr Recibido: 4 de marzo-2007 - Aprobado: 30 de abril-2007
} 
pública por parte de las comunidades indígenas de Chirripó. Para ello, hace un llamado a representantes comunales y a las instituciones con presencia en estos territorios e integra la Comisión Siwä Pakö. En este proceso de reflexión, acuerda respetar el derecho humano de los pueblos originarios de ser educados desde su diferencia, a pesar de las limitaciones estructurales, espaciales e ideológicas que, históricamente, han determinado el desarrollo educativo en esta región.

Como paralelo a este proceso, se plantea desarrollar, desde la Acción Social, el Proyecto Jornadas de auto y mutua capacitación con los maestros y las maestras del Programa de Lengua y Cultura del distrito de Chirripó, con el propósito de perfilar procesos educativos culturalmente pertinentes.

El proceso de globalización tiende a difuminar las fronteras políticas y territoriales y con ellas, las identidades nacionales andadas en los discursos de la modernidad y el imaginario de Estado-Nación. En este contexto, el proceso de diferenciación cultural, lejos de eliminarse, se hará más evidente ante la posibilidad de comparamos con los otros, cada vez más cercanos y cotidianos en este continuo evolutivo, que trae una mayor conciencia de las diferencias entre entidades culturales, tanto en lo interno como en lo externo de las naciones.

Este proceso ejerce una fuerte presión sobre la cultura en sus niveles estructurales y funcionales, repercutiendo en la complejidad de los diversos términos de los colectivos, como jerarquización, distribución de riqueza, poder, valores, creencias y todo lo que relacionado con ellos se aprende en cada grupo social, con una historicidad y memoria cultural.

La cultura adquiere diversas formas a través del tiempo y del espacio. Esta diversidad se manifiesta en la originalidad y la pluralidad de las identidades que caracterizan los grupos y sociedades que componen la humanidad.

Considerando que la diversidad cultural debe ser fuente de intercambio, de innovación, de creatividad, un derecho humano inalienable, lejos de ello, se han procurado procesos históricos de marginación y exclusión social.

\section{Antecedentes}

La sede del Atlántico integra en el año 2004, una comisión interinstitucional al tenor de la resolución "Políticas Estratégicas: admisión, permanencia y graduación", emitida por el VI Congreso Universitario en el que se reconoce el deber de la Universidad de Costa Rica de promover el acceso a la educación superior en condiciones de equidad y sin discriminación a estudiantes de recursos limitados en todo el país, considerando además que su área de 
influencia cuenta con una población aproximada de cinco mil personas de la etnia cabécar de Chirripó.

La población cabécar es un grupo indígena costarricense, que habita el territorio ubicado en diferentes puntos de la Cordillera de Talamanca. El territorio está dividido en dos secciones, una en la provincia de Limón y otra en la de Cartago, siendo esta última la del área de influencia de la sede del Atlántico.

En la búsqueda de alternativas para cumplir con este mandato institucional, se inicia un proceso reflexivo que ha permitido definir, en compañia de representantes de este grupo humano y de organizaciones e instituciones estatales con presencia en territorios indígenas,-una serie de acciones para convertir la educación en un verdadero motor del desarrollo regional y permitir a la población cabécar, la participación activa en la construcción de nuevas oportunidades de desarrollo.

Al respecto, Watson et al. (2006) señalan que:

... "es una realidad que en el Territorio Cabécar, la educación no ha jugado ese papel por razones diversas gue han servido de excusa pero que son ampliamente cuestionables desde los derechos culturales de los pueblos originarios: lo remoto y lo extenso de su territorio, el reducido tamaño de su población, el monolingüismo o desconocimiento del español, la ausencia de vías de comunicación y servicios públicos básicos entre otros aspectos" (p. 1).

Lo expuesto anteriormente es confirmado con el hecho de que a pesar de que la sede del Atlántico cumplió en el año 2006, treinta y cinco años de fundación, no hay registros de ingreso a la institución de una persona de la comunidad cabécar.

\section{Proyecto: Jornadas de auto y mutua capacitación con los maestros y las maestras de Lengua y Cultura}

\section{Justificación}

La primera escuela en el territorio cabécar se crea en la década de los años ochenta, lo que supone la negación hasta este momento, del derecho constitucional a la educación pública, por parte del Estado a este grupo de la población costarricense. Este tardío desarrollo de la educación en la región, aunado al dificil acceso de las comunidades a los centros educativos, hacen 
que se conciba la escuela como una instittición ajena a su cultura. Asimismo, la ausencia de personas nativas calificadas para el ejercicio docente es un factor que incide en el estado actual de los procesos educativos que se desarrollan en estas escuelas.

Es aquí donde se inscribe esta propuesta de mejoramiento de las competencias docentes de los maestros y las maestras indígenas que se desempeñan desde el Programa de Lengua y Cultura del Ministerio de Educación Pública. Este proyecto parte de la visión de la escuela como instrumento social que permite la identificación y cultivo del patrimonio natural y sociocultural de este grupo humano, de modo que la visión de mundo de las personas cabécares sea asumida en el espacio escolar, lo que implica que esté anclada en su contexto, en su cultura, pero a la vez, proyectada al país y al mundo, perfilando desde procesos educativos culturalmente pertinentes, competencias integrales que les permita insertarse al desarrollo social y laboral.

Con el propósito de incidir en el mejoramiento de la práctica docente de los maestros y las maestras del Programa de Lengua y Cultura, se planteó el desarrollo de jornadas de auto y mutua capacitación, como una alternativa en el corto plazo, mientras que en el mediano plazo las instancias responsables diseñan y ejecutan acciones estratégicas para solventar estas particularidades educativas.

Esta propuesta de auto y mutua capacitación impulsa la construcción de una actitud dialógica, el modelaje pedagógico y la incorporación de la interculturalidad, como fundamentos para el abordaje crítico de la didáctica específica del ámbito cabécar y el mejoramiento de los procesos educativos que se desarrollan en las escuelas de este territorio.

\section{Metodología}

El proyecto se desarrolla mediante diversas técnicas de trabajo grupal tales como: charlas, talleres, cine-foros, entre otras, en una sesión mensual de ocho horas de duración. Se realizan ocho sesiones al año.

Para la programación de las diferentes actividades, se consideran los resultados de un diagnóstico de necesidades e intereses temáticos que se realiza previamente con la población participante.

\section{Objetivos}

El objetivo general para el proyecto es: Promover el reconocimiento de las características socioculturales de las personas de los territorios indígenas 
del distrito Chirripó -Circuito 06- y considerar esta información para generar procesos de aprendizaje más favorables y significativos.

Como objetivos específicos se proponen:

- Analizar la pertinencia cultural presente o no en los procesos de enseñanza-aprendizaje de las escuelas indígenas.

- Determinar el grado de conocimientos básicos que poseen los y las docentes de Lengua y Cultura, con respecto de la pedagogía, la didáctica y el currículo.

- Posibilitar espacios de observación de la labor pedagógica a este grupo de docentes en contextos educativos similares y diferentes.

- Promover círculos de estudio y reflexión con las y los docentes de Lengua y Cultura, con respecto a las temáticas de atención prioritaria, según los resultados del diagnóstico.

- Caracterizar elementos de la cosmovisión de los cabécares de Chirripó, sobre el ambiente, la educación, la salud, la familia, el trabajo, la organización comunitaria y su lengua, susceptibles de ser incorporados en el trabajo de aula.

\section{Logros}

En los dos años de ejecución de este proyecto, se han logrado impactos significativos tales como:

- La vinculación entre la sede del Atlántico y el Departamento de Educación Indígena del Ministerio de Educación Pública, que permite el trabajo conjunto.

- La asistencia constante de la mayoría de los maestros y las maestras de Lengua y Cultura a las sesiones de trabajo, a pesar de la lejanía y dificultad que significa trasladarse a la sede del Atlántico.

- La oportunidad para los maestros y las maestras de Lengua y Cultura de contar con la posibilidad de una actualización y reflexión permanentes sobre temas relacionados con el ejercicio de su práctica docente, que han permitido:

- la articulación de los procesos de enseñanza-aprendizaje en correspondencia con el entorno natural, sociocultural y personal, 
- la formulación de orientaciones pedagógicas de carácter intercultural para la incorporación de la cultura cabécar en el espacio del aula escolar,

- la promoción de correspondencia y coherencia de los procesos educativos con la lengua cabécar como una forma de valorarla y mantenerla,

- el abordaje crítico de la diversidad de los procesos culturales en la escuela, para valorar las culturas y generar actitudes de respeto por el otro e interacciones enriquecedoras que hagan de la escuela un lugar democrático, solidario y equitativo.

\section{Consideraciones finales}

El Proyecto Jornadas de auto y mutua capacitación con los maestros y las maestras de Lengua y Cultura, en sus dos años de desarrollo, ha dado respuesta a una necesidad sentida por los maestros y maestras del Programa de Lengua y Cultura, puesto que se ha ejecutado en una relación dialógica de trabajo. Esto ha permitido impactar positivamente la práctica pedagógica de las escuelas indígenas. 


\section{REFERENCIAS}

Universidad de Costa Rica. (2005). Proyecto ED - 1764 Jornadas de auto y mutua capacitación a maestros y maestras de Lengua y Cultura.

Watson, H., Bolaños, M., Brenes, C. (2006). La escuela desde el territorio indígena cabécar de Chirripó, Costa Rica. Universidad de Costa Rica: Memoria III Jornadas de Divulgación "La visión del desarrollo sostenible a través de cuatro cristales". Costa Rica. 